\title{
Avaliação ultrassonográfica da anomalia vascular portossistêmica em cão: relato de caso
}

\author{
[Ultrasonographic evaluation of portosystemic vascular anomaly in dog: a case report] \\ M.A.R. Feliciano ${ }^{1}$, C.A.L. Leite $^{2}$, A.C. Nepomuceno ${ }^{3}$, T. Silveira ${ }^{4}$, \\ R.A.L. $M u z z i^{2}$, W.R.R. Vicente ${ }^{5}$ \\ ${ }^{1}$ Aluno de pós-graduação - FCAV-UNESP - Jaboticabal, SP \\ ${ }^{2}$ Departamento de Medicina Veterinária - UFLA - Lavras, MG \\ ${ }^{3}$ Médica veterinária residente - UFLA - Lavras, MG \\ ${ }^{4}$ Médica veterinária autônoma - Lavras/MG \\ ${ }^{5}$ Faculdade de Ciências Agrárias e Veterinárias - UNESP - Jaboticabal, SP
}

\begin{abstract}
RESUMO
Relata-se a avaliação ultrassonográfica, com auxílio do Doppler em cores, da anomalia vascular portossistêmica em um cão da raça Poodle Toy, que apresentou edema pulmonar e alterações gastrintestinais. Ao exame ultrassonográfico, foram observados vasos portais dilatados e tortuosos. Esses resultados associados aos achados clínicos e laboratoriais permitem identificar a anomalia vascular portossistêmica no paciente.
\end{abstract}

Palavras-chave: cão, malformação vascular, fístula portossistêmica, ultrassom

\begin{abstract}
It was reported the ultrasonographic evaluation, assisted to Doppler color, of the portosystemic vascular anomaly in a Poodle Toy dog. The dog was referred with lung edema and gastrointestinal alterations. At ultrasonographic exam, portal vessels were extended and tortuous. These findings associated to clinical and laboratorial findings, allowed to identify the vascular portosystemic anomaly in the patient.
\end{abstract}

Keywords: dog, vascular malformation, portosystemic fistula, ultrasound

\section{INTRODUÇÃO}

A ultrassonografia pode ser utilizada para avaliar uma variedade de doenças invasivas e nãoinvasivas que afetam o parênquima hepático dos animais (Nyland e Park, 1983; Johnson, 2003).

As anormalidades vasculares podem ser detectadas por exame ultrassonográfico, com possibilidades de diagnóstico da anomalia vascular portossistêmica (AVPS) (Nyland e Park, 1983; Johnson, 2003). Dentre as alterações vasculares que interferem na fisiologia hepática, são descritas as anomalias vasculares portossistêmicas, que podem ser intra ou extrahepáticas e as fístulas arteriovenosas (Johnson, 2003; Fox et al., 2004; Leveille-Webster, 2004). Os desvios intra-hepáticos são classificados de acordo com sua localização no órgão e sua forma de apresentação (Lamb e White, 1998).

A anomalia vascular portossistêmica é uma malformação venosa que provoca anastomose da circulação sistêmica ao sistema porta, permitindo que o sangue portal desvie do fígado (circulação hepatofugal). Essa alteração pode ser intra ou extra-hepática e, geralmente, apenas um vaso é acometido (Johnson, 2003). No entanto, existem relatos de animais que apresentam desvios portossistêmicos intra e extra-hepáticos concomitantes.

A circulação hepatofugal elimina os metabólitos do sistema hepatocelular do sangue portal, que contêm toxinas derivadas do intestino. Dessa forma, essa alteração pode causar o

Recebido em 30 de junho de 2008

Aceito em 9 de março de 2009

E-mail: marcusfeliciano@yahoo.com.br 
desenvolvimento episódico de encefalopatia hepática associada à ingestão de alimentos ricos em proteína, sangramento gastrintestinal, azotemia, distúrbios eletrolíticos, transfusão sanguínea, infecções e administração de certos fármacos. Os sinais neurológicos estão relacionados à encefalopatia hepática, que promove alterações comportamentais, ataxia, alterações da visão, convulsões e andar em círculo. A perfusão hepática torna-se comprometida e a ausência de fatores hepatotrópicos resulta em micro-hepatia. Em seguida, ocorre hiperamonemia e déficit na transformação de ácido úrico à alantoína, causando cristalúria ou urolitíase amoniacal. $\mathrm{Na}$ urinálise encontram-se hipostenúria e cristalúria de biurato de amônio, substância que em condições normais seria biotransformada pelo fígado. Os sinais gastrintestinais observados são anorexia, ptialismo, vômito, diarreia, polifagia e disfagia. Animais com esta alteração apresentam peso geralmente abaixo do normal. Também foram verificados casos de ascite (Uchino et al., 1999; Sassaki et al., 2001; Ferrell et al., 2003; Johnson, 2003). Um dos diferenciais para AVPS é a displasia microvascular hepatoportal (Schermerhorn et al., 1996).

A incidência da AVPS é maior nos cães de raças puras (Australian Cattle Dog, Cairn Terrier, Golden Retriever, Irish Wolfhound, Maltês, Old English Sheepdog, Poodle, Pug, Retriever do Labrador, Schnauzer Miniatura, Shih Tzu, Teckel e Yorkshire Terrier) e gatos mestiços, havendo hipótese de predisposição racial genética. Por ser uma alteração congênita, os animais com até um ano de idade são os mais acometidos, apesar de haver relatos de cães que apresentaram os primeiros sintomas com até 10 anos de idade (Hunt et al., 2000; Sassaki et al., 2001; Johnson, 2003; Tobias e Rohrbach, 2003).

A prevalência da AVPS é desconhecida, sendo relatada apenas na América do Norte, Japão, Inglaterra e Austrália (Johnson, 2003).

Ao exame ultrassonográfico podem ser visibilizadas as anomalias vasculares que envolvem a veia porta e a vasculatura sistêmica. Em geral, os desvios intra-hepáticos são mais fáceis de visibilizar que os desvios extrahepáticos (Leveille-Webster, 2004). Entretanto, algumas fístulas portossistêmicas intra-hepáticas podem não ser visibilizadas devido às limitações impostas pela micro-hepatia e gases do trato gastrintestinal (Nyland et al., 1995). Os desvios portossistêmicos extra-hepáticos adquiridos são secundários à hipertensão portal, que pode ser de causa pré-hepática, hepática ou pós-hepática. Doença hepática crônica e cirrose são enfermidades que podem gerar hipertensão portal como causa hepática. Esta anomalia vascular caracteriza-se ao exame ultrassonográfico pela visibilização de múltiplos pequenos vasos tortuosos, com origem na porção extra-hepática da veia porta ou em suas tributárias, se estendendo à região renal, podendo fazer conecção com a veia renal e gonadal (Park et al., 1990; Nyland et al., 1995; Howe et al., 2000).

Por meio da ultrassonografia é visibilizada micro-hepatia subjetiva, hipervascularidade de vasos hepáticos, desvio e dilatação de vasos hepáticos e estruturas vasculares portais alteradas (Johnson, 2003; Koide et al., 2004). Na fístula extra-hepática, os ramos portais intra-hepáticos geralmente são de difícil visibilização, sugerindo hipovascularização.

A utilização do Doppler colorido aumenta a capacidade do examinador de detectar os desvios intra e extra-hepáticos e também é útil neste diagnóstico (Gallego et al., 2002; LeveilleWebster, 2004). Carvalho e Chammas (2008) citam a visibilização ao Doppler colorido de um vaso anômalo, geralmente tortuoso, e com fluxo turbulento em casos de fístula portossistêmica.

Um dos métodos de diagnóstico recentemente descrito na literatura humana é a ultrassonografia tridimensional, a qual permite avaliação de melhores detalhes anatômicos vasculares, como dilatações e tortuosidades (Oguz et al., 2003). Imagens de tomografia computadorizada podem mostrar a comunicação entre a veia portal e a veia hepática, bem como assimetrias no trajeto da veia hepática (Gallego et al., 2002).

O tratamento é baseado na estabilização clínica sintomática do paciente, incluindo uma dieta controlada, sendo que, na maioria das vezes, é necessária uma correção cirúrgica (Park et al., 1990).

O objetivo deste trabalho foi relatar a detecção ultrassonográfica, com auxílio do Doppler colorido, de anomalia vascular portossistêmica em um cão da raça Poodle Toy. 


\section{CASUÍSTICA}

Um cão da raça Poodle Toy, com dois anos de idade e pesando $5,0 \mathrm{~kg}$, apresentou tosse seca e episódios de dispneia desde filhote. Nesta época, foi radiografado e instaurou-se diagnóstico sugestivo de colapso de traqueia e doença pulmonar obstrutiva crônica. $\mathrm{O}$ animal foi tratado e apresentou melhora clínica.

Após um ano de terapia, voltou a apresentar os episódios de tosse seca e dispneia. Ao exame físico, o animal apresentou estado geral bom. À palpação abdominal externa, foi verificado o espessamento de alças intestinais com presença de conteúdo líquido. Foi repetida a radiografia torácica, com padrão sugestivo de edema pulmonar. Foi instituída terapia para controle desse edema e do quadro de tosse. O proprietário relatou que o animal apresentou um episódio de diarreia a partir de então.

$\mathrm{Na}$ última consulta e após avaliação clínica, foram realizadas dosagens de fosfatase alcalina (FA), alanina aminotransferase (ALT) e bilirrubina direta (BD). Foi indicado o exame ultrassonográfico para avaliação de trato gastrintestinal e fígado. Também foram realizados exames ecoDopplercardiográfico e radiográfico.

\section{DISCUSSÃO}

A avaliação bioquímica sérica para este paciente apontou aumento de ALT (360UI/1) e manutenção dos níveis séricos de FA e bilirrubina dentro dos valores normais de referência para a espécie. Dados de literatura sugerem que a avaliação bioquímica dos animais com AVPS fornece baixa dosagem de ureia, creatinina, glicose e colesterol, além de atividade variável de ALT e FA (geralmente elevadas em pacientes jovens) e nível de BD dentro dos valores de referência (Johnson, 2003). Esse autor atribui estes achados laboratoriais normais à lesão de atrofia hepática ou à mínima lesão hepatocelular.

Durante auscultação pulmonar, foi notada presença de crepitação fina discreta e difusa em lobo pulmonar esquerdo. À ausculta cardíaca, foi detectado sopro mesossistólico em valva mitral de grau I/VI. Ao exame
ecoDopplercardiográfico, foram verificados aumento discreto de ventrículo esquerdo, valva mitral e tricúspide com cúspides espessadas em grau moderado e discreto, respectivamente. Notou-se a presença de refluxo turbulento sistólico indicativo de insuficiência valvar leve em ambas as valvas. A valva aórtica apresentou folhetos espessados em grau discreto, sugestivo de calcificação. No entanto, todos estes sinais de doença degenerativa mixomatosa de valvas mitral e tricúspide não apresentam qualquer relação com o quadro de AVPS, constituindo um achado incidental. Segundo Johnson (2003), o edema pulmonar observado se relaciona com a hipoalbuminemia e não com achados de insuficiência cardíaca. Porém, na presença de sopro mesossistólico e sinais de crepitações pulmonares, um exame ecocardiográfico torna-se imprescindível para o diagnóstico de exclusão.

Ao exame radiográfico torácico laterolateral esquerdo, foram visibilizados assimetria de traqueia e padrão pulmonar misto (intersticial e bronquial), sugerindo a presença de colapso de traqueia e edema pulmonar. De acordo com a literatura, alguns animais com AVPS apresentam edema pulmonar moderado e quadro de ascite, devido à hipoalbuminemia, condizendo com os achados da radiografia torácica (Johnson, 2003). $\mathrm{O}$ quadro de tosse seca e de caráter transitório se confirma na presença radiográfica do colapso de traqueia.

Confirmando os achados ultrassonográficos citados por alguns autores, foram visibilizados vasos portais dilatados e tortuosos (Fig. 1), sugerindo o quadro de anomalia vascular portossistêmica, intra-hepática (Johnson, 2003; Leveille-Webster, 2004). De acordo com a literatura, o exame ultrassonográfico apresenta $91 \%$ e $84 \%$ de sensibilidade em cães e gatos, respectivamente, para o diagnóstico de AVPS (D'Anjou et al., 2004).

Por meio do Doppler colorido, foi avaliada a presença da anomalia vascular e a turbulência dos vasos (Fig. 2). De acordo com Kudo et al. (1993), as imagens por Doppler colorido demonstram uma comunicação de fluxo entre a veia portal e a veia hepática, observando-se também turbulência sanguínea. 


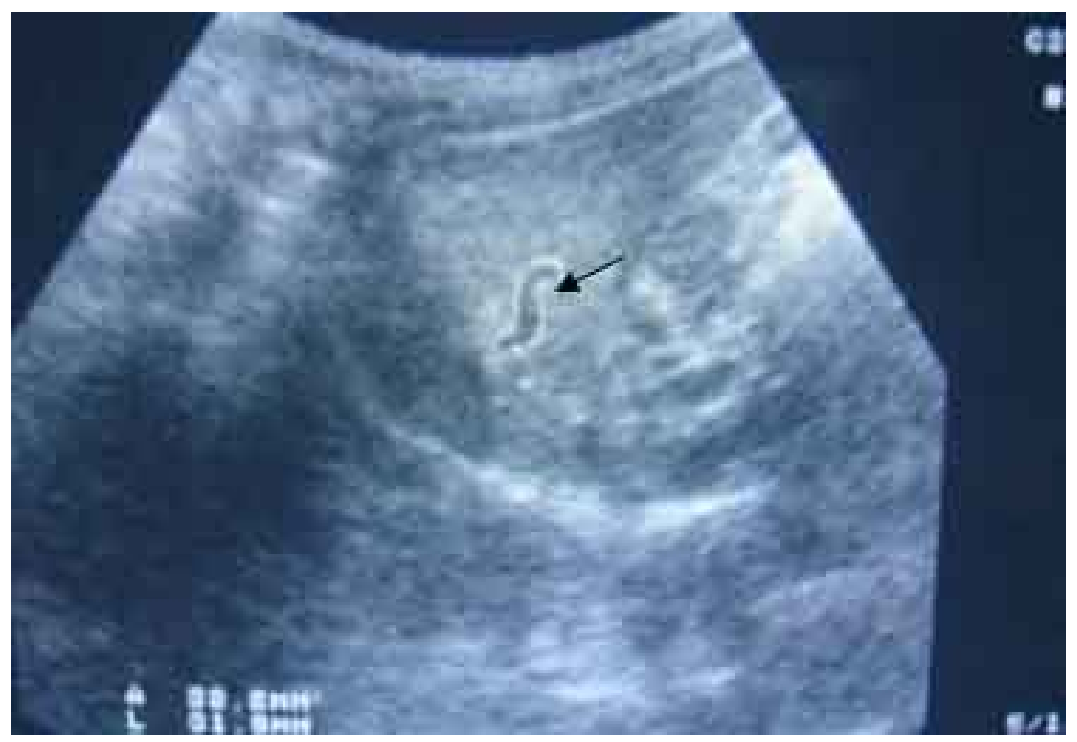

Figura 1. Imagem ultrassonográfica do fígado de um cão da raça Poodle Toy, de dois anos de idade. A seta indica a presença de um vaso tortuoso e calibroso (área de $38,2 \mathrm{~mm}^{2}$ ), sugerindo a presença da anomalia vascular portossistêmica.

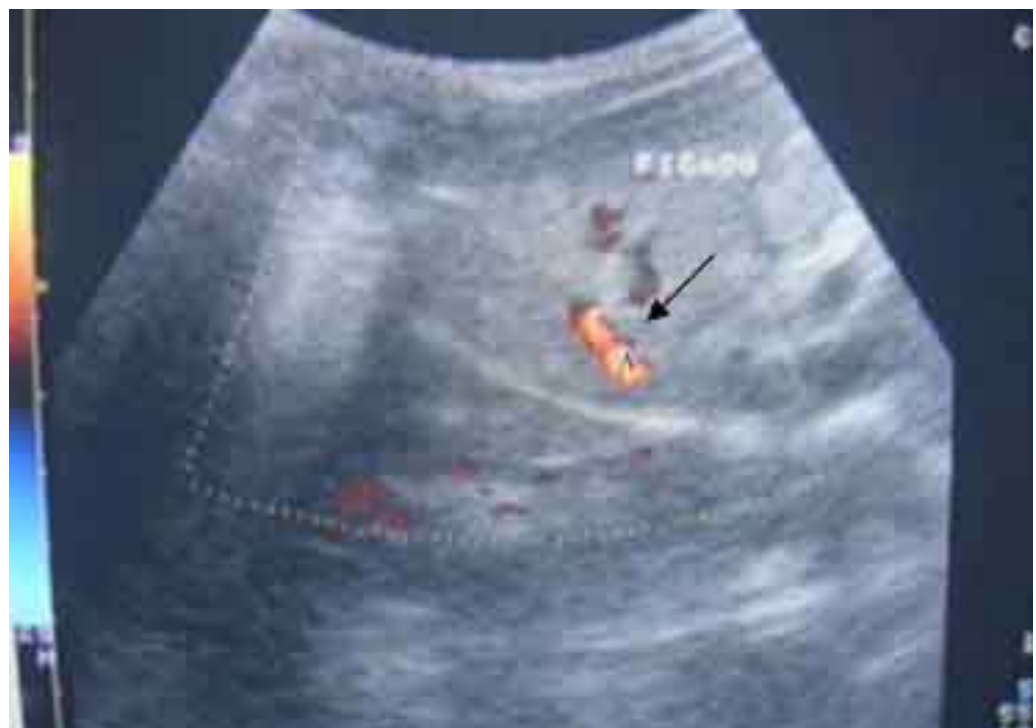

Figura 2. Imagem Doppler em cores do fígado de um cão da raça Poodle Toy, de dois anos de idade. A anomalia vascular e seu fluxo sanguíneo correspondente (A) são apontados pela seta.

\section{CONCLUSÕES}

Por meio da avaliação ultrassonográfica, associada aos achados clínicos e de exames laboratoriais, foi possível a detecção de anomalia vascular portossistêmica no paciente, sendo este o primeiro relato de anomalia vascular portossistêmica no Brasil. Há na literatura veterinária nacional apenas relatos de fístulas portossistêmicas (shunts).

\section{REFERÊNCIAS BIBLIOGRÁFICAS}

CARVALHO, C.F.; CHAMMAS, M.C. Uso do ultrassom duplex Doppler no diagnóstico de shunt portossistêmico em gatos. Arq. Bras. Med. Vet. Zootec., v.60, p.109-112, 2008.

D'ANJOU, M.A.; PENNINCK, D.; CORNEJO, L. et al. Ultrasonographic diagnosis of portosystemic shunting in dogs and cats. Vet. Radiol. Ultrasound, v.45, p.424-437, 2004. 
FERRELL, E.A.; GRAHAM, J.P.; HANEL, R. et al. Simultaneous congenital and acquired extrahepatic portosystemic shunts in two dogs. Vet. Radiol. Ultrasound, v.44, p.38-42, 2003.

FOX, P.R.; PETRIE, J.; SUTER, P.F. Doença vascular periférica. In: ETTINGER, S.J.; FELDMAN, E.C. Tratado de medicina interna veterinária. Doenças do cão e do gato. 5.ed. Rio de Janeiro: Guanabara-Koogan, 2004. p.1026.

GALLEGO, C.; VELASCO, M.; MARCUELLO, P. et al. Congenital and acquired anomalies of the portal venous system. Radiographics, v.22, p.141-159, 2002.

HOWE, L.M.; BOOTHE JR., H.W.; MILLER, M.W. et al. A canine model of multiple portosystemic shunting. J. Invest. Surg., v.13, p.45-57, 2000.

HUNT, G.B.; TISDALL, P.L.C.; WEBB, A. et al. Congenital portosystemic shunts in Toy and Miniature Poodles. Aust. Vet. J., v.78, p.530-532, 2000.

JOHNSON, S.E. Desvio sanguíneo portossistêmico. In: TILLEY, L.P.; SMITH JR., F.W.K. Consulta veterinária em 5 minutos. Espécie canina e felina. 2.ed. São Paulo: Manole, 2003. p.1108-1109.

KOIDE, K.; KOIDE, Y.; WADA, Y. et al. Congenital hepatic arteriovenous fistula with intrahepatic portosystemic shunt and aortic stenosis in a dog. J. Vet. Med. Sci., v.66, p.299$302,2004$.

KUDO, M.; TOMITA, S.; TOCHIO, H. et al. Intrahepatic portosystemic venous shunt: diagnosis by color Doppler imaging. Am. J. Gastroenterol., v.88, p.723-729, 1993.

LAMB，C.R.; WHITE, R.N. Morphology of congenital intrahepatic portacaval shunts in dogs and cats. Vet. Rec., v.142, p.55-60, 1998.

LEVEILLE-WEBSTER, C.R. Diagnóstico laboratorial de doenças hepatobiliares. In:
ETTINGER, S.J.; FELDMAN, E.C. Tratado de medicina interna veterinária. Doenças do cão e do gato. 5.ed. Rio de Janeiro: GuanabaraKoogan, 2004. p.1363-1398.

NYLAND, T.G.; PARK, R.D. Hepatic ultrasonography in the dog. Vet. Radiol. Ultrasound, v.24, p.74, 1983.

NYLAND, T.G.; MATOON, J.S.; WISNER, E.R. Ultrasonography of the liver. In: MATTOON, J.S.; NYLAND, T.G. Veterinary diagnostic ultrasound. Londres: W.B. Saunders, 1995. p.70-73.

OGUZ, B.; AKATA, D.; BALKANCI, F. et al. Intrahepatic portosystemic venous shunt: diagnosis by colour/power Doppler imaging and three-dimensional ultrasound. $B r . J$. Radiol., v.76, p.487-490, 2003.

PARK, J.H.; CHA, S.H.; HAN, J.K. et al. Intrahepatic portosystemic venous shunt. Am. J. Roentgenol., v.155, p.527-528, 1990.

SASSAKI, R.A.; SASSAKI, E.L.; SASSAKI, M.S. Desvio portossistêmico congênito simples extra-hepático: correção com constritor ameróide. Relato de caso. Clin. Vet., v.7, p.2732,2001 .

SCHERMERHORN, T.; CENTER, S.A.; DYKES, N.L. et al. Characterization of hepatoportal microvascular dysplasia in a kindred of cairn terriers. J. Vet. Int. Med., v.10, p.219-230, 1996.

TOBIAS, K.M.; ROHRBACH, B.W. Association of breed with the diagnosis of congenital portosystemic shunts in dogs: 2400 cases (1980-2002). J. Am. Vet. Med. Assoc., v.223, p.1636-1639, 2003.

UCHINO, T.; MATSUDA, I.; ENDO, F. The long-term prognosis of congenital portosystemic venous shunt. J. Pediatr., v.135, p.254-256, 1999. 